\title{
Studies on the Synthesis of Vitamin D Analogs with Aromatic D-Ring
}

\author{
SILVINA EDUARDO-CANOSA, MARÍA MARCO, RITA SIGÜEIRO and ANTONIO MOURIÑO
}

Laboratorio de Investigación Ignacio Ribas, Departamento de Química Orgánica, Universidad de Santiago de Compostela, Avda. Ciencias, s/n, 15782 Santiago de Compostela, Spain

Manuscript received on September 6, 2017; accepted for publication on October 10, 2017

\begin{abstract}
Herein, we describe our studies on the synthesis of $1 \alpha, 25$-dihydroxyvitamin $\mathrm{D}_{3}$ analogs possessing a benzene ring replacing the natural 5-membered $\mathbf{D}$-ring by the Wittig-Horner and dienyne approaches. A key feature is the synthesis of a $\mathrm{Cr}(\mathrm{CO})_{3}$-complexed previtamin $\mathrm{D}$ derivative that enables the construction of vitamin D analogs with aromatic D-ring through a thermal [1,7]-H sigmatropic shift. This study establishes the basis for the design of new vitamin $\mathrm{D}$ analogs containing aromatic $\mathbf{D}$-ring, complexed or uncomplexed to $\mathrm{Cr}(\mathrm{CO})_{3}$ type moieties for specific molecular recognition and drug research and development.
\end{abstract}

Key words: D-ring modified vitamin D analogs, aryl- $\mathrm{Cr}(\mathrm{CO})_{3}$ complexes, cancer, sigmatropic rearrangements, synthesis.

\section{INTRODUCTION}

1 $\alpha, 25$-Dihydroxyvitamin $\mathrm{D}_{3}[1,1,25 \mathrm{D}$, calcitriol, Figure 1], the most potent steroid hormone known and the active metabolite of the seco-steroid vitamin $\mathrm{D}_{3}$, interacts with the vitamin $\mathrm{D}$ nuclear receptor (VDR), a member of the nuclear receptor superfamily, to dimerize with the retinoid $\mathrm{X}$ receptor (RXR). The heterodimer binds to the vitamin D response elements(VDREs) in target gene promoters and recruit coactivator proteins to induce a cascade of events including control of mineral homeostasis and various important cellular processes such as differentiation, anti-proliferation, growth, angiogenesis, apoptosis, and immunomodulation

Correspondence to: Antonio Mouriño

E-mail: antonio.mourino@usc.es

* Contribution to the centenary of the Brazilian Academy of Sciences.
(Norman et al. 1979, Evans et al. 1988, Kliewer et al. 1992, Bouillon et al. 1995, Feldman et al. 2011). Recently, it was discovered that $1,25 \mathrm{D}$ activates more than 229 genes associated with several diseases, including arthritis, diabetes and cancer, suggesting that this hormone might have an even broader range of biological functions than originally thought (Lincoln et al. 2010) 1,25D itself has clinical applications, but the pharmacological doses required for treatment of cancer induce strong, undesired hypercalcemia (Plum et al. 2010, Feldman et al. 1997). Structure-function studies of numerous $1,25 \mathrm{D}$ analogs have shown that the undesired calcemic effects can be reduced during cell-differentiating activities, but the mechanism of this selectivity has not yet been unraveled (Feldman et al. 2011, Glebocka et al. 2012). Increasing synthetic efforts have been directed at the 

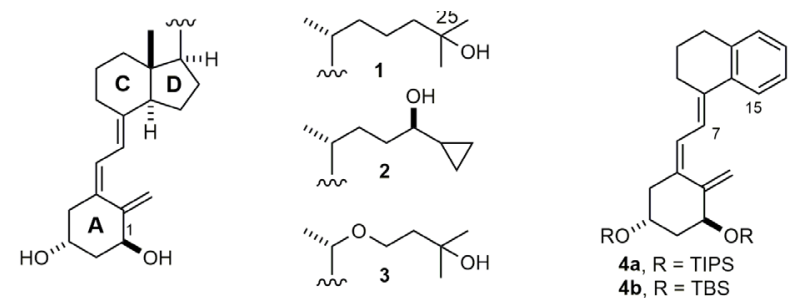

Figure 1 - Structures of 1,25D (1), calcipotriol (2), OCT (3) and target D-ring-aromatic compound 4.

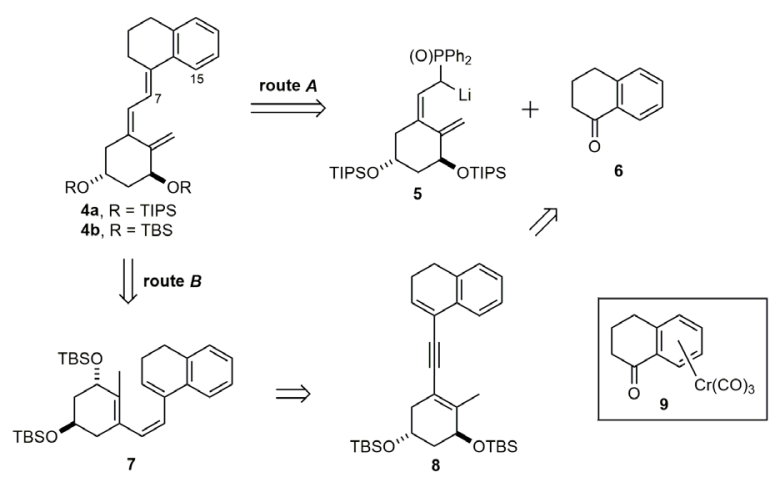

Figure $\mathbf{2}$ - Retrosynthesis of target $\mathbf{4}$ by the Wittig-Horner and Dienyne approaches.

development of non-calcemic analogs of the natural hormone 1,25D for treatment of specific disorders, but only a few have found clinical applications. Among these, calcipotriol (2, Figure 1) and OCT (3), two analogs with structural modifications at the side chain, are being successfully used for treatment of psoriasis (Feldman et al. 1997). A few structural features that reduce the calcemic activity include: a) unsaturations at the side chain or D-ring (Verlinden et al. 2000), b) the lack of the 19-methylene group (Kensler et al. 2000), c) 14-epi-configuration (Verlinden et al. 2000, Ma et al. 2013), d) 3-epi-configuration (Molnar et al. 2011), e) short non-hydroxylated side chains (Plum et al. 2004), f) CD-carboranic mimics (Yamada et al. 2014) and g) $o$-carboranic side chains (Otero et al. 2016). Nonsecosteroidal vitamin D receptor modulators that reduce calcemic effects have also been reported (Eduardo-Canosa et al. 2010).

Our continued interest in the synthesis of $1,25 \mathrm{D}$ analogs with selective properties as potential drugs for treatment of cancer and/or psoriasis led us to study the synthesis of aromatic compounds $4 \mathbf{a}$ and $\mathbf{4 b}$ (Figure 1) as models, which bear a benzene ring replacing the natural 5-membered D-ring. Preliminary studies to build the triene system of 4 utilizing the Wittig-Horner coupling approach (route $\boldsymbol{A}$ ) (Lythgoe 1980) between phosphineoxide-lithium anion 5 and ketone $\mathbf{6}$ resulted in the recovery of starting ketone presumably through the corresponding enolate (Figure 2). Attempts to synthesize $\mathbf{4 b}$ through the dienyne approach (route $\boldsymbol{B}$ ) (Castedo et al. 1986) were hampered by difficulties to accomplish the sigmatropic $[1,7]-\mathrm{H}$ shift on the previtamin 7 , presumably due to crossconjugation of the triene system with the aromatic ring. Here we describe our efforts to synthesize 4a through route $\boldsymbol{A}$ and $\mathbf{4 b}$ through route $\boldsymbol{B}$, from ketone- $\mathrm{Cr}(\mathrm{CO})_{3}$ complex 9. Complexation with $\mathrm{Cr}(\mathrm{CO})_{6}$ was envisioned as a way to reduce conjugation of the aromatic ring with either the carbonyl group in tetralone $\mathbf{6}$ or the triene system in 7 .

\section{MATERIALS AND METHODS}

\section{GENERAL MATERIALS AND METHODS}

Reagents were purchased from Aldrich Chemical (www.sigma-aldrich.com) or Acros Organics (www.acros.com) and used without further purification. All reactions involving oxygen or moisture sensitive compounds were carried out under dry argon atmosphere using oven-dried or flame-dried glassware and standard syring/septa tecniques. All dry solvents were distilled under argon immediately prior to use: Tetrahydrofuran (THF), $\mathrm{Et}_{2} \mathrm{O}$ and $n-\mathrm{Bu}_{2} \mathrm{O}$ were distilled from $\mathrm{Na} /$ benzophenone. $\mathrm{CH}_{2} \mathrm{Cl}_{2}$ and $\mathrm{Ac}_{2} \mathrm{O}$ were distilled from $\mathrm{P}_{2} \mathrm{O}_{5}$. Hexanes, $n$-heptane, diisopropilamine $\left(i-\mathrm{PrNH}_{2}\right)$ and triethylamine $\left(\mathrm{Et}_{3} \mathrm{~N}\right)$ were distilled from $\mathrm{CaH}_{2}$. $\mathrm{MeOH}$ was distilled from $\mathrm{Mg} / \mathrm{I}_{2}$. DMSO was distilled from $\mathrm{CaH}_{2}$ and stored over activated $4 \AA$ molecular sieves. Solutions of $n$-butyllithium 
in hexanes were titrated with $\mathrm{N}$-benzylbenzamide before use. Hexanes refer to a distilled mixture of hexane isomers. Reaction temperatures refer to external bath temperatures. Acetone-dry ice baths were used for reactions at low temperature. Alternatively, acetone baths were cooled with a CRYOCOOL immersion cooler, provided with a temperature regulator. Organic extracts were dried over anhydrous $\mathrm{Na}_{2} \mathrm{SO}_{4}$, filtered and concentrated using rotary evaporator at aspirator pressure $(20-30 \mathrm{mmHg})$. Sat refers to aqueous saturated solution. Reactions were monitored by thin-layer chromatography (TLC) using aluminum-backed Merck 60 silica gel plates ( $0.2 \mathrm{~mm}$ thickness). After visualization under ultraviolet light at $254 \mathrm{~nm}$, the plates were developed by immersion in a solution containing either a mixture of $p$-anisaldehyde (2.5\%), acetic acid (1\%), and sulfuric acid (3.4\%) in $95 \%$ ethanol or a solution of ceric ammonium nitrate $(0.5 \mathrm{~g})$ and ammonium molybdate $(4.8 \mathrm{~g})$ in $\mathrm{H}_{2} \mathrm{O}(100 \mathrm{~mL})$ and $\mathrm{H}_{2} \mathrm{SO}_{4}(5.6 \mathrm{~mL})$ followed by heating with a heater gun. Flash column chromatography was performed with Merck silica gel (230-400 mesh). HPLC purifications were performed on a Shimadzu preparative liquid chromatograph, model LC- $8 \mathrm{~A}$, equipped with a TSP 1100 UV absorbance detector using a HPLC Phenomenex-Luna silica column (Ø 25 x $250 \mathrm{~mm}$ $5 \AA \sim 10 \mathrm{~mm}$ ). NMR spectra were recorded in $\mathrm{CDCl}_{3}$ solutions on a Bruker AMX $500 \mathrm{MHz}$, Varian Inova $400 \mathrm{MHz}$ or Bruker DPX $250 \mathrm{MHz}$. Chemical shifts are reported on the $\delta$ scale (ppm) downfield from tetramethylsilane $(\delta=0.0 \mathrm{ppm})$ using the residual solvent signal at $\delta=7.26 \mathrm{ppm}\left({ }^{1} \mathrm{H}, \mathrm{CDCl}_{3}\right)$ or $\delta=77.0 \mathrm{ppm}\left({ }^{13} \mathrm{C}, \mathrm{t}, \mathrm{CDCl}_{3}\right)$ as internal standard; coupling constants $(J)$ are reported in $\mathrm{Hz}$. Distortionless enhancement by polarization transfer (DEPT-135) was used to assign carbon types. Low (MS) and high resolution mass spectra (HRMS) were performed in a Micromas Instruments Autospec spectrometer.

\section{SYNTHESIS}

1,2,3,4-Tetrahydronaphthalen-1-ol (10). $\mathrm{NaBH}_{4}$ ( $0.850 \mathrm{~g}, 22.45 \mathrm{mmol}, 1.16$ equiv) was added in one portion to a $-78^{\circ} \mathrm{C}$ cooled solution of ketone 6 (2.83 g, $19.35 \mathrm{mmol}, 1$ equiv) in $\mathrm{MeOH}(85 \mathrm{~mL})$. The mixture was allowed to reach $23{ }^{\circ} \mathrm{C}$ and then concentrated in vacuo. The residue was dissolved in $\mathrm{CH}_{2} \mathrm{Cl}_{2}(40 \mathrm{~mL})$ and washed with sat $\mathrm{NaCl}$ (30 $\mathrm{mL})$. The organic phase was dried, filtered and concentrated in vacuo. The residue was purified by flash chromatography (10\% EtOAc/hexanes) to give alcohols $\mathbf{1 0}$ (Zhao et al. 2012) [2.810 g, $18.96 \mathrm{mmol}, 98 \%$, colorless oil, $\mathrm{R}_{f}=0.56(40 \%$ EtOAc/hexanes)]. ${ }^{1} \mathrm{H}-\mathrm{NMR}\left(250 \mathrm{MHz}, \mathrm{CDCl}_{3}\right): \delta$ $7.46(1 \mathrm{H}, \mathrm{m}), 7.25(2 \mathrm{H}, \mathrm{m}), 7.16(1 \mathrm{H}, \mathrm{m}), 4.78(1 \mathrm{H}$, m), $2.81(2 \mathrm{H}, \mathrm{m}), 2.33(1 \mathrm{H}, \mathrm{s}), 2.11-1.73(4 \mathrm{H}, \mathrm{m})$. ${ }^{13} \mathrm{C}-\mathrm{NMR}\left(63 \mathrm{MHz}, \mathrm{CDCl}_{3}\right): \delta 138.7(\mathrm{C}), 137.0$ (C), $128.8(\mathrm{CH}), 128.6(\mathrm{CH}), 127.4(\mathrm{CH}), 126.0$ $(\mathrm{CH}), 67.9(\mathrm{CH}, \mathrm{C}-1), 32.1\left(\mathrm{CH}_{2}\right), 29.1\left(\mathrm{CH}_{2}\right), 18.7$ $\left(\mathrm{CH}_{2}\right)$.

Tricarbonyl $\left[\eta^{6}-(1,2,3,4-\right.$ tetrahydronaftalen1-ol)] chromium(0) (11). Alcohol 10 (0.100 g, 0.67 mmol, 1.5 equiv) and $\mathrm{Cr}(\mathrm{CO})_{6}(0.222 \mathrm{~g}, 1.01$ mmol, 1 equiv) were dissolved in a mixture of $n$ - $\mathrm{Bu}_{2} \mathrm{O} / n$-heptane $(8 \mathrm{~mL}, 1: 1)$. The solution was deoxygenated. A stream of argon was passed through the solution. Dry THF $(0.4 \mathrm{~mL})$ was added. The reaction mixture was heated at reflux in the dark for $54 \mathrm{~h}$. The mixture was allowed to reach $23{ }^{\circ} \mathrm{C}$ and filtered through a pad of celite. The solids were washed with $\mathrm{CH}_{2} \mathrm{Cl}_{2}$ and the combined solution was concentrated in vacuo. The residue was purified by flash chromatography $(50 \%$ $\mathrm{CH}_{2} \mathrm{Cl}_{2} /$ hexanes) to give $\mathbf{1 1}$ (Schmalz et al. 1992) [74 mg, $0.26 \mathrm{mmol}, 39 \%$, yellow solid, $\mathrm{R}_{f}=0.20$ (EtOAc/ $\mathrm{CH}_{2} \mathrm{Cl}_{2} /$ hexanes, 1:10:10)] and starting material 10 [40 mg, $0.27 \mathrm{mmol}, 40 \%$ ]. ${ }^{1} \mathrm{H}-\mathrm{NMR}$ $\left(250 \mathrm{MHz}, \mathrm{CDCl}_{3}\right): \delta 5.81(1 \mathrm{H}, \mathrm{d}, J=6.5 \mathrm{~Hz})$, $5.49(1 \mathrm{H}, \mathrm{t}, J=6.2 \mathrm{~Hz}), 5.09(2 \mathrm{H}, \mathrm{m}), 4.49(1 \mathrm{H}$, $\mathrm{m}), 2.84-2.50$ (2H, m), 2.23-1.85 (2H, m), 1.79$1.61(2 \mathrm{H}, \mathrm{m}) .{ }^{13} \mathrm{C}-\mathrm{NMR}\left(63 \mathrm{MHz}, \mathrm{CDCl}_{3}\right): \delta 233.3$ 
$\left(3 \times \mathrm{C}, \mathrm{Cr}(\mathrm{CO})_{3}\right), 113.8(\mathrm{C}), 112.7(\mathrm{C}), 95.1(\mathrm{CH})$, $93.4(\mathrm{CH}), 90.1(\mathrm{CH}), 88.9(\mathrm{CH}), 66.6(\mathrm{CH}, \mathrm{C}-1)$, $32.2\left(\mathrm{CH}_{2}\right), 27.5\left(\mathrm{CH}_{2}\right), 19.2\left(\mathrm{CH}_{2}\right)$.

Tricarbonyl- $\left[\eta^{6}-(3,4-d i h y d r o n a f t a l e n-\right.$ 1(2H)-one)] chromium(0) (9). A solution of alcohol $11(0.600 \mathrm{~g}, 2.11 \mathrm{mmol})$ in $\mathrm{Ac}_{2} \mathrm{O}(9 \mathrm{~mL})$ and DMSO $(13.5 \mathrm{~mL})$ was stirred at $23{ }^{\circ} \mathrm{C}$ for $3.5 \mathrm{~h}$. The reaction mixture was poured into a 0 ${ }^{\circ} \mathrm{C}$ cooled solution of $\mathrm{NaOH}(250 \mathrm{~mL}, 10 \%)$. The resulting mixture was extracted with $\mathrm{Et}_{2} \mathrm{O}(3 \times 30$ $\mathrm{mL})$. The combined organic layers were dried, filtered and concentrated in vacuo. The residue was purified by flash chromatography [EtOAc/ $\mathrm{CH}_{2} \mathrm{Cl}_{2}$ /hexanes $\left.(1: 10: 10)\right]$ to give ketone 9 $\left[0.500 \mathrm{~g}, 1.77 \mathrm{mmol}, 84 \%\right.$, orange solid, $\mathrm{R}_{f}=0.44$ (EtOAc $/ \mathrm{CH}_{2} \mathrm{Cl}_{2} /$ hexanes, 1:10:10)]. ${ }^{1} \mathrm{H}-\mathrm{NMR}(250$ $\left.\mathrm{MHz}, \mathrm{CDCl}_{3}\right): \delta 6.15(1 \mathrm{H}, \mathrm{d}, J=6.6 \mathrm{~Hz}), 5.62$ $(1 \mathrm{H}, \mathrm{t}, J=6.1 \mathrm{~Hz}), 5.28(1 \mathrm{H}, \mathrm{t}, J=6.3 \mathrm{~Hz}), 5.14$ $(1 \mathrm{H}, \mathrm{d}, J=5.4 \mathrm{~Hz}), 2.95(1 \mathrm{H}, \mathrm{m}), 2.71(2 \mathrm{H}, \mathrm{dt}$, $\left.J_{1}=16.8 \mathrm{~Hz}, J_{2}=4.1 \mathrm{~Hz}\right), 2.43(1 \mathrm{H}, \mathrm{m}), 2.12(2 \mathrm{H}$, m). ${ }^{13} \mathrm{C}-\mathrm{NMR}\left(63 \mathrm{MHz}, \mathrm{CDCl}_{3}\right): \delta 230.7(3 \mathrm{xC}$, $\left.\mathrm{Cr}(\mathrm{CO})_{3}\right), 196.0(\mathrm{CO}, \mathrm{C}-1), 115.4(\mathrm{C}), 94.8(\mathrm{CH})$, $92.6(\mathrm{C}), 91.2(\mathrm{CH}), 89.8(\mathrm{CH}), 89.2(\mathrm{CH}), 37.7$ $\left(\mathrm{CH}_{2}\right), 28.3\left(\mathrm{CH}_{2}\right), 21.6\left(\mathrm{CH}_{2}\right)$.

(1E)-Tricarbonyl- $\left\{\eta^{6}-1,2,3,4-t e t r a h y d r o-1-\right.$ [(Z)-2-((3S,5S)-3,5-bis-[(triisopropilsilyl)oxy]-2methylenecyclohexyliden)ethyliden]-naftalen\} chromium(0) (12). A solution of $n$-BuLi in hexanes ( $0.69 \mathrm{~mL}, 1.46 \mathrm{mmol}, 2.1 \mathrm{M}, 2.7$ equiv) was slowly added to a $-78{ }^{\circ} \mathrm{C}$ cooled solution of 5 (1.10 g, $1.65 \mathrm{mmol}, 3$ equiv) in dry THF $(20 \mathrm{~mL})$. After $1 \mathrm{~h}$, a solution of 9 ( $0.155 \mathrm{~g}, 0.55 \mathrm{mmol}, 1$ equiv) in dry THF ( $8 \mathrm{~mL})$ was added via cannula. The reaction mixture was allowed to reach $15{ }^{\circ} \mathrm{C} . \mathrm{H}_{2} \mathrm{O}$ $(0.5 \mathrm{~mL})$ were added. The mixture was concentrated in vacuo. The residue was dissolved in $\mathrm{Et}_{2} \mathrm{O}$ (30 $\mathrm{mL}$ ) and successively washed with sat $\mathrm{NaHCO}_{3}$ $(30 \mathrm{~mL})$ and sat $\mathrm{NaCl}(30 \mathrm{~mL})$. The combined aqueous layers were re-extracted with $\mathrm{Et}_{2} \mathrm{O}(3 \times 20$ $\mathrm{mL})$. The combined organic extracts were dried, filtered and concentrated in vacuo. The residue was purified by flash chromatography $(10 \%$
EtOAc/hexanes) to give a mixture of compounds 12 and $\mathbf{1 3}$ (40 mg) and the starting material 9 (99 $\mathrm{mg}, 0.35 \mathrm{mmol}, 64 \%$ ). The mixture of $\mathbf{1 2}$ and $\mathbf{1 3}$ was purified by preparative HPLC (hexanes) to afford four diasteroisomers: 12a (3 mg, $4 \mu \mathrm{mol}$, 0.7\%), 12b (24 mg, $0.03 \mathrm{mmol}, 5.5 \%)$, 13a (2 mg, $2.7 \mu \mathrm{mol}, 0.5 \%)$ and $\mathbf{1 3 b}$ (7 mg, $0.01 \mathrm{mmol}, 1.7 \%$ ). Compound 12b: ${ }^{1} \mathrm{H}-\mathrm{NMR}\left(500 \mathrm{MHz}, \mathrm{CDCl}_{3}\right): \delta$ $6.96(1 \mathrm{H}, \mathrm{d}, J=11.1 \mathrm{~Hz}), 6.24(1 \mathrm{H}, \mathrm{d}, J=11.1$ $\mathrm{Hz}), 5.60(1 \mathrm{H}, \mathrm{m}), 5.45(1 \mathrm{H}, \mathrm{s}), 5.30-5.24(3 \mathrm{H}$, m), $5.00(1 \mathrm{H}, \mathrm{s}), 4.60(1 \mathrm{H}, \mathrm{m}), 4.37(1 \mathrm{H}, \mathrm{m}), 2.78$ $\left(1 \mathrm{H}, \mathrm{dt}, J_{1}=9.2 \mathrm{~Hz}, J_{2}=4.4 \mathrm{~Hz}\right), 2.68-2.53(3 \mathrm{H}$, m), 2.43-2.30 (2H, m), $2.08(1 \mathrm{H}, \mathrm{m}), 1.93-1.82$ $(3 \mathrm{H}, \mathrm{m}), 1.15-1.03$ (42H, m). ${ }^{13} \mathrm{C}-\mathrm{NMR}(75 \mathrm{MHz}$, $\left.\mathrm{CDCl}_{3}\right): \delta 233.5\left(3 \mathrm{xC}, \mathrm{Cr}(\mathrm{CO})_{3}\right), 149.2(\mathrm{C}), 142.3$ (C), $128.8(\mathrm{C}), 122.5(\mathrm{CH}), 122.4(\mathrm{CH}), 111.8$ $\left(\mathrm{CH}_{2}\right), 109.5(\mathrm{C}), 106.6(\mathrm{C}), 93.8(\mathrm{CH}), 91.5(\mathrm{CH})$, $91.2(\mathrm{CH}), 87.1(\mathrm{CH}), 71.1(\mathrm{CH}), 67.8(\mathrm{CH}), 46.1$ $\left(\mathrm{CH}_{2}\right), 45.1\left(\mathrm{CH}_{2}\right), 29.4\left(\mathrm{CH}_{2}\right), 25.7\left(\mathrm{CH}_{2}\right), 22.0$ $\left(\mathrm{CH}_{2}\right), 18.2\left(6 \mathrm{xCH}_{3}\right), 18.1\left(6 \mathrm{xCH}_{3}\right), 12.4(3 \mathrm{xCH})$, $12.3(3 \mathrm{xCH}) . \mathrm{MS}\left([\mathrm{CI}]^{+}, \mathrm{m} / \mathrm{z}, \%\right): 730\left([\mathrm{M}]^{+}, 10\right)$, $687\left(\left[\mathrm{M}-{ }^{i} \mathrm{Pr}\right]^{+}, 32\right), 594\left(\left[\mathrm{M}-\mathrm{Cr}(\mathrm{CO})_{3}\right]^{+}, 70\right), 557$ ([M-OTIPS $\left.]^{+}, 84\right), 247$ [M-H-2xOTIPS-Cr(CO) $]^{+}$, 100). HMRS ([CI $\left.]^{+}\right)$: calcd for: $\left[\mathrm{C}_{40} \mathrm{H}_{62} \mathrm{CrO}_{5} \mathrm{Si}_{2}\right]^{+}$ ([M] $\left.]^{+}\right)$: 730.3541; found: 730.3550. Compound 13b: ${ }^{1} \mathrm{H}-\mathrm{NMR}\left(500 \mathrm{MHz}, \mathrm{CDCl}_{3}\right): \delta 6.45(1 \mathrm{H}, \mathrm{d}, J$ $=11.2 \mathrm{~Hz}), 6.40(1 \mathrm{H}, \mathrm{d}, J=11.1 \mathrm{~Hz}), 5.79(1 \mathrm{H}, \mathrm{d}, J$ $=6.3 \mathrm{~Hz}), 5.40(1 \mathrm{H}, \mathrm{s}), 5.35(1 \mathrm{H}, \mathrm{m}), 5.28(2 \mathrm{H}, \mathrm{m})$, $4.99(1 \mathrm{H}, \mathrm{s}), 4.59(1 \mathrm{H}, \mathrm{m}), 4.37(1 \mathrm{H}, \mathrm{m}), 2.69(2 \mathrm{H})$, $2.58(1 \mathrm{H}, \mathrm{d}, J=13.0 \mathrm{~Hz}), 2.45(1 \mathrm{H}, \mathrm{m}), 2.39(2 \mathrm{H}$, m), $2.01(2 \mathrm{H}, \mathrm{m}), 1.89(1 \mathrm{H}, \mathrm{m}), 1.81(1 \mathrm{H}, \mathrm{m}), 1.16$ 0.99 (42H, m). ${ }^{13} \mathrm{C}-\mathrm{NMR}\left(75.47 \mathrm{MHz}, \mathrm{CDCl}_{3}\right): \delta$ 233.3 (3xC, $\left.\mathrm{Cr}(\mathrm{CO})_{3}\right), 148.6$ (C), 141.9 (C), 130.9 (C), $126.2(\mathrm{CH}), 123.5(\mathrm{CH}), 112.1\left(\mathrm{CH}_{2}\right), 110.3$ (C), $107.0(\mathrm{C}), 94.1(\mathrm{CH}), 92.6(\mathrm{CH}), 92.5(\mathrm{CH})$, $90.1(\mathrm{CH}), 71.0(\mathrm{CH}), 67.8(\mathrm{CH}), 45.8\left(\mathrm{CH}_{2}\right), 45.0$ $\left(\mathrm{CH}_{2}\right), 34.0\left(\mathrm{CH}_{2}\right), 28.2\left(\mathrm{CH}_{2}\right), 23.2\left(\mathrm{CH}_{2}\right), 18.2$ $\left(6 \mathrm{xCH}_{3}\right), 18.1\left(6 \mathrm{xCH}_{3}\right), 12.4(3 \mathrm{xCH}), 12.3(3 \mathrm{xCH})$.

Tricarbony $1-\left\{\eta^{6}-[3,4-d\right.$ i h y d ro- 1 ((trifluoromethanesulfonyl)oxy)naftalen]\} chromium(0) (14). 
A solution of lithium diisopropylamide was prepared by slowly addition of a solution of $n$-BuLi in hexanes $(0.42 \mathrm{~mL}, 0.92 \mathrm{mmol}, 2.2 \mathrm{M}, 1.3$ equiv) to a-78 ${ }^{\circ} \mathrm{C}$ cooled neat $i-\mathrm{Pr}_{2} \mathrm{NH}(0.14 \mathrm{~mL}, 1.01$ mmol, 1.4 equiv). The bath was removed and the white slurry was stirred at $23{ }^{\circ} \mathrm{C}$ for $15 \mathrm{~min}$. The suspension was cooled to $-78^{\circ} \mathrm{C}$ and dry THF (2.5 $\mathrm{mL}$ ) was added. After $15 \mathrm{~min}$, a solution of ketone 9 $(0.200 \mathrm{~g}, 0.71 \mathrm{mmol}, 1$ equiv) in dry THF $(2 \mathrm{~mL})$ was added dropwise. The reaction mixture was stirred at $-78{ }^{\circ} \mathrm{C}$ for $45 \mathrm{~min}$ and a solution of $N, N-(5-$ chloropyridin-2-yl)-bistriflimide (0.444 g, 1.13 mmol, 1.6 equiv $)$ in dry THF $(1.5 \mathrm{~mL})$ was added via cannula. The mixture was allowed to reach $23{ }^{\circ} \mathrm{C}$. The reaction was quenched by the addition of $\mathrm{H}_{2} \mathrm{O}$ $(10 \mathrm{~mL})$. The mixture was extracted with EtOAc ( $3 \times 10 \mathrm{~mL})$. The combined organic layers were dried, filtered and concentrated. The residue was purified by flash chromatography (10\% EtOAc/ hexanes) to give $\mathbf{1 4}[0.215 \mathrm{~g}, 0.52 \mathrm{mmol}, 73 \%$, orange oil, $\mathrm{R}_{f}=0.44(20 \%$ EtOAc/hexanes $\left.)\right]$. ${ }^{1} \mathrm{H}-\mathrm{NMR}\left(250 \mathrm{MHz}, \mathrm{CDCl}_{3}\right): \delta 6.02(1 \mathrm{H}, \mathrm{m}), 5.55$ $(1 \mathrm{H}, \mathrm{m}), 5.12-5.38(3 \mathrm{H}, \mathrm{m}), 2.85(1 \mathrm{H}, \mathrm{m}), 2.40-$ $2.72(3 \mathrm{H}, \mathrm{m}) .{ }^{13} \mathrm{C}-\mathrm{NMR}\left(63 \mathrm{MHz}, \mathrm{CDCl}_{3}\right): \delta 232$ $\left(3 \mathrm{xC}, \mathrm{Cr}(\mathrm{CO})_{3}\right), 124.3(\mathrm{CH}), 120.1(\mathrm{C}), 107.5$ (C), $95.7(\mathrm{C}), 92.0(\mathrm{CH}), 91.4(\mathrm{CH}), 89.9(\mathrm{CH})$, $86.7(\mathrm{CH}), 25.6\left(\mathrm{CH}_{2}\right), 21.5\left(\mathrm{CH}_{2}\right)$. MS $\left([\mathrm{CI}]^{+}\right.$, $\mathrm{m} / \mathrm{z}, \%): 415\left([\mathrm{M}+\mathrm{H}]^{+}, 97\right), 414\left([\mathrm{M}]^{+}, 98\right), 279$ $\left(\left[\mathrm{M}+\mathrm{H}-\mathrm{Cr}(\mathrm{CO})_{3}\right]^{+}, 43\right), 266\left([\mathrm{M}+\mathrm{H}-\mathrm{OTf}]^{+}, 96\right), 147$ (100). HMRS ([CI $]^{+}$): calcd for: $\left[\mathrm{C}_{14} \mathrm{H}_{10} \mathrm{O}_{6} \mathrm{~F}_{3} \mathrm{SCr}\right]^{+}$ $\left([\mathrm{M}+\mathrm{H}]^{+}\right): 414.9555$; found: 414.9559 .

Tricarbonyl- $\left\{\eta^{6}-(1,2-d i h y d r o-4-[(3 S, 5 R)-\right.$ 3,5-bis-(tert-butyld imethylsilyloxy)-2 methyl-cyclohex-1-en-1-yl]ethynyl)-naftalen chromium(0) (16). Compound 15 (0.120 g, 0.31 mmol, 1.3 equiv), $\mathrm{PdCl}_{2}\left(\mathrm{PPh}_{3}\right)_{2}(8 \mathrm{mg}, 0.011 \mathrm{mmol}$, $5 \mathrm{~mol} \%)$ and $\mathrm{CuI}$ ( $2 \mathrm{mg}, 0.011 \mathrm{mmol}, 5 \mathrm{~mol} \%)$ were successively added to a solution of $\mathbf{1 4}(0.100$ $\mathrm{g}, 0.24 \mathrm{mmol}, 1$ equiv) in dry $\mathrm{Et}_{3} \mathrm{~N}(3 \mathrm{~mL})$. The reaction mixture was heated at $60{ }^{\circ} \mathrm{C}$ for $2 \mathrm{~h}$. The mixture was allowed to reach $23{ }^{\circ} \mathrm{C}$ and then filtered through a pad of celite. The solids were washed with $\mathrm{CH}_{2} \mathrm{Cl}_{2}(3 \times 5 \mathrm{~mL})$ and the combined solution was washed with a sat $\mathrm{NH}_{4} \mathrm{Cl}(20 \mathrm{~mL})$. The aqueous layer was extracted with $\mathrm{CH}_{2} \mathrm{Cl}_{2}(3 \times 25 \mathrm{~mL})$. The combined organic extracts were dried, filtered and concentrated in vacuo. The residue was purified by flash chromatography (hexanes) to give $\mathbf{1 6}$ [0.121 g, $0.187 \mathrm{mmol}, 78 \%$, orange oil, $\mathrm{R}_{f}=0.60(20 \%$ EtOAc/hexanes)]. ${ }^{1} \mathrm{H}-\mathrm{NMR}\left(250 \mathrm{MHz}, \mathrm{CDCl}_{3}\right): \delta$ $6.43\left(1 \mathrm{H}, \mathrm{dd}, J_{1}=6.0 \mathrm{~Hz}, J_{2}=3.7 \mathrm{~Hz}\right), 5.82(1 \mathrm{H}$, $\left.\mathrm{dd}, J_{1}=6.0 \mathrm{~Hz}, J_{2}=2.2 \mathrm{~Hz}\right), 5.45-5.20(3 \mathrm{H}, \mathrm{m})$, $4.24(1 \mathrm{H}, \mathrm{m}), 4.14(1 \mathrm{H}, \mathrm{m}), 2.86-2.35(5 \mathrm{H}, \mathrm{m})$, $2.14(1 \mathrm{H}, \mathrm{m}), 1.98(3 \mathrm{H}, \mathrm{s}), 1.87(1 \mathrm{H}, \mathrm{m}), 1.72(1 \mathrm{H}$, m), $0.92(9 \mathrm{H}, \mathrm{s}), 0.90(9 \mathrm{H}, \mathrm{s}), 0.12(6 \mathrm{H}$, br s), 0.08 $(6 \mathrm{H}, \mathrm{s}) \cdot{ }^{13} \mathrm{C}-\mathrm{NMR}\left(63 \mathrm{MHz}, \mathrm{CDCl}_{3}\right): \delta 233.2(3 \mathrm{xC}$, $\left.\mathrm{Cr}(\mathrm{CO})_{3}\right), 142.6(\mathrm{C}), 136.7(\mathrm{CH}), 118.9(\mathrm{C}), 114.7$ (C), $107.5(\mathrm{C}), 102.2(\mathrm{C}), 92.5(\mathrm{CH}), 92.1(\mathrm{C}), 91.5$ $(\mathrm{CH}), 91.2(\mathrm{CH}), 90.7(\mathrm{CH}), 87.2(\mathrm{C}), 69.8(\mathrm{CH})$, $64.1(\mathrm{CH}), 41.1\left(\mathrm{CH}_{2}\right), 39.4\left(\mathrm{CH}_{2}\right), 26.0\left(\mathrm{CH}_{2}\right)$, $25.9\left(3 \mathrm{xCH}_{3}\right), 25.8\left(3 \mathrm{xCH}_{3}\right), 23.4\left(\mathrm{CH}_{2}\right), 19.3$ $\left(\mathrm{CH}_{3}\right), 18.1(\mathrm{C}), 18.0(\mathrm{C}),-4.3\left(\mathrm{CH}_{3}\right),-4.6\left(\mathrm{CH}_{3}\right)$, $-4.7\left(\mathrm{CH}_{3}\right),-4.8\left(\mathrm{CH}_{3}\right) . \mathrm{MS}\left([\mathrm{CI}]^{+}, \mathrm{m} / \mathrm{z}, \%\right): 644$ $\left([\mathrm{M}]^{+}, 13\right), 560\left([\mathrm{M}-3 \mathrm{xCO}]^{+}, 8\right), 377$ ([M-OTBS$\left.\left.\mathrm{Cr}(\mathrm{CO})_{3}\right]^{+}, 83\right), 245\left[\mathrm{M}-\mathrm{H}-2 \mathrm{TBSO}-\mathrm{Cr}(\mathrm{CO})_{3}\right]^{+}$, 100). HMRS ([CI $\left.]^{+}\right)$: calcd for: $\left[\mathrm{C}_{34} \mathrm{H}_{48} \mathrm{CrO}_{5} \mathrm{Si}_{2}\right]^{+}$ $\left([\mathrm{M}]^{+}\right)$: 644.2445 ; found: 644.2448 .

Tricarbonyl- $\left\{\eta^{6}-[1,2-d i h y d r o-4 Z-[(3 S, 5 R)-\right.$ 3,5-bis-(tert-butyldimethylsilyloxy)-2-methylciclohexen-1-yl)vinyl]-naftalen chromium(0) (17). A suspension of quinoleine in hexanes (50 $\mathrm{mL}, 2 \mathrm{mmol}, 0.04 \mathrm{M}, 1.25$ equiv) and Lindlar catalyst (167 mg, $\mathrm{Pd} / \mathrm{CaCO}_{3}$ poisoned with lead) were successively added to a solution of enyne $\mathbf{1 6}$ ( $0.100 \mathrm{~g}, 0.16 \mathrm{mmol}, 1$ equiv) in dry hexanes (27 $\mathrm{mL}$ ). The system was purged three times with argon and two times with hydrogen. The reaction mixture was stirred under hydrogen at balloon pressure for $1 \mathrm{~h}$ and then filtered through a pad of celite. The solids were washed with hexanes and the combined solution was concentrated in vacuo. The residue was purified by flash chromatography (hexanes) to give 16 [0.036 g, $0.06 \mathrm{mmol}, 35 \%$, red oil, $\mathrm{R}_{f}=0.70$ (20\% EtOAc/hexanes)]. ${ }^{1} \mathrm{H}-\mathrm{NMR}$ (250 
$\left.\mathrm{MHz}, \mathrm{CDCl}_{3}\right): \delta 6.24(1 \mathrm{H}, \mathrm{d}, J=12.1 \mathrm{~Hz}), 6.04$ $(1 \mathrm{H}$, br s), 5.49-5.19 (5H, m), 4.32-3.85 $(2 \mathrm{H}, \mathrm{m})$, 2.90-1.20 (11H, m), $0.88(18 \mathrm{H}, \mathrm{m}), 0.05(12 \mathrm{H}, \mathrm{m})$. MS $\left([\mathrm{CI}]^{+}, \mathrm{m} / \mathrm{z}, \%\right): 510\left(\left[\mathrm{M}-\mathrm{Cr}(\mathrm{CO})_{3}\right]^{+}, 20\right)$, 379 ([M-Cr(CO) $\left.\left.)_{3}-\mathrm{OTBS}\right]^{+}, 86\right), 355$ (49), 247 $\left([\mathrm{M}-\mathrm{H}-2 \mathrm{TBSO}]^{+}, 100\right)$. HMRS $\left([\mathrm{CI}]^{+}\right)$: calcd for: $\left[\mathrm{C}_{34} \mathrm{H}_{50} \mathrm{CrO}_{5} \mathrm{Si}_{2}\right]^{+}\left([\mathrm{M}]^{+}\right): 646.2602$; found: 646.2626 .

$(1 R, 3 S, 5 Z)-5-[(E)-2-(2,3-D i h y d r o n a p h t h a l e n-$ 4(1H)-yliden)ethylidene]-1,3-bis-(tert-butyl dimethylsilyloxi)-4-methylenecyclohexane (4) and $(1 R, 3 S)-5-[(Z)-2-(1,2-d i h y d r o n a p h t h a l e n-$ 4-yl)vinyl]-1,3-bis-(tert-butyldimethylsilyloxi)4-methyl-4-cyclohexene (7). A solution of $\mathbf{1 7}$ $(0.030 \mathrm{~g}, 0.046 \mathrm{mmol})$ in dry isooctane $(5 \mathrm{~mL})$ was heated at reflux for $2 \mathrm{~h}$. The reaction was allowed to reach $23{ }^{\circ} \mathrm{C}$ and then concentrated in vacuo. The residue was re-dissolved in $\mathrm{MeOH}$ ( $3 \mathrm{~mL}$ ). The resulting solution was cooled at $0{ }^{\circ} \mathrm{C}$ and a solution of CAN [ammonium cerium(IV) nitrate] in $\mathrm{MeOH}$ $(1.5 \mathrm{~mL})$ was added until green color remained. The mixture was extracted with hexanes $(3 \times 5$ $\mathrm{mL})$. The combined organic extract was washed with $\mathrm{H}_{2} \mathrm{O}(10 \mathrm{~mL})$, dried, filtered and concentrated in vacuo. The residue was purified by flash chromatography (5\% EtOAc/hexanes) to afford a mixture of compounds $\mathbf{4 b}$ and 7 [0.016 g, 0.031 mmol, $68 \%$, ca 1:0.7, colorless oil, $\mathrm{R}_{f}=0.70(5 \%$ EtOAc/hexanes)]. ${ }^{1} \mathrm{H}-\mathrm{NMR}\left(250 \mathrm{MHz}, \mathrm{CDCl}_{3}\right): \delta$ 7.54 (H15-4b, d, $J=7.7 \mathrm{~Hz}$ ), 7.22-7.04 (Hs-Ar, br m), 6.99 (H7-4b, d, $J=11.3 \mathrm{~Hz}$ ), 6.34 (H6-4b, d, $J=11.3 \mathrm{~Hz}), 6.21(\mathrm{H} 7+\mathrm{H} 6-7, \mathrm{~d}, J=12.2 \mathrm{~Hz}), 5.98$ (H9-7, t, $J=4.7$ Hz), 5.31 (H19-4b, s), 4.95 (H194b, d, $J=2.2$ Hz), 4.46 (H1-4b, m), 4.30-3.89 (H34b, H1-7, H3-7, m), 2.88-1.50 (Hs-4+7, m), 0.930.78 ( $t$-BuSi + Me19-7, m), 0.14-0.03 (MeSi, m).

\section{RESULTS AND DISCUSSION}

Complex 9 was prepared according to Schmalz's procedure (Figure 3) (Schmalz et al. 1992). Reduction of ketone $\mathbf{6}$ with sodium borohydride in methanol provided alcohol $\mathbf{1 0}$ in $98 \%$ yield. Heating at reflux a mixture of $\mathbf{1 0}$ and chromium hexacarbonyl in $n$-heptane $/ n-\mathrm{Bu}_{2} \mathrm{O} / \mathrm{THF}(10: 10: 1)$ gave the diastereomeric alcohols 11 in 39\% yield, which upon oxidation with $\mathrm{Ac}_{2} \mathrm{O}$ and DMSO produced the desired $\mathrm{Cr}(\mathrm{CO})_{3}$-complex 9 in $84 \%$ yield. Wittig-Horner coupling between ketone 9 and phosphine oxide anion 5 in THF furnished a mixture of four diastereomers $(\mathbf{1 2}+\mathbf{1 3})$, which could be separated by HPLC [12 (two diastereomers $0.7 \%+$ $5.5 \%$ ); 13 (two distereomers $0.5+1.7 \%$ )] together with recovered starting ketone 9 (64\%). Each pair of diastereomers could not be distinguished by ${ }^{1} \mathrm{H}$ NMR (500 MHz). The H7 and H15 of 12 appear deshielded with respect to those corresponding to $\mathbf{1 3}$ (steroid numbering is used for discussion). The structure 12 was established by ${ }^{1} \mathrm{H}$ NMR nOe analysis.

Though the $\mathrm{Cr}(\mathrm{CO})_{3}$-complexation reduces conjugation of the aromatic unit with the carbonyl group, the yield on Wittig-Horner reaction products is still too low to considerer this approach of preparative value. This result led us to examine the dienyne approach (route $\boldsymbol{B}$, Figure 2) as an alternative pathway to the target compound $\mathbf{4 b}$ (Figure 4). Treatment of ketone 9 with LDA followed trapping of the resulting enolate with 2-[N,N-bis(trifluoromethylsulfonyl)-amino]-5chloropyridine afforded vinyl triflate 14 (73\%), which upon Sonogashira coupling (Sonogashira et al. 1998) with enyne 15 in the presence catalytic amounts of $\mathrm{CuI}$ and $\mathrm{PdCl}_{2}\left(\mathrm{PPh}_{3}\right)_{2}$ provided the dienyne 16 (68\%). Semihydrogenation of the triple bond in the presence of Lindlar catalyst and quinoline in hexanes gave the previtamin D 17 in $35 \%$ yield. Finally, thermal sigmatropic $[1,7]-$ $\mathrm{H}$ shift on 17 in refluxing isooctane followed by $\mathrm{Cr}(\mathrm{CO})_{3}$-decomplexation with ammonium cerium(IV) nitrate in methanol gave a 1:0.7 mixture of the target protected vitamin $\mathbf{4 b}$ and the corresponding previtamin 7 in $68 \%$ yield. The formation of the previtamin 7 can be rationalized 


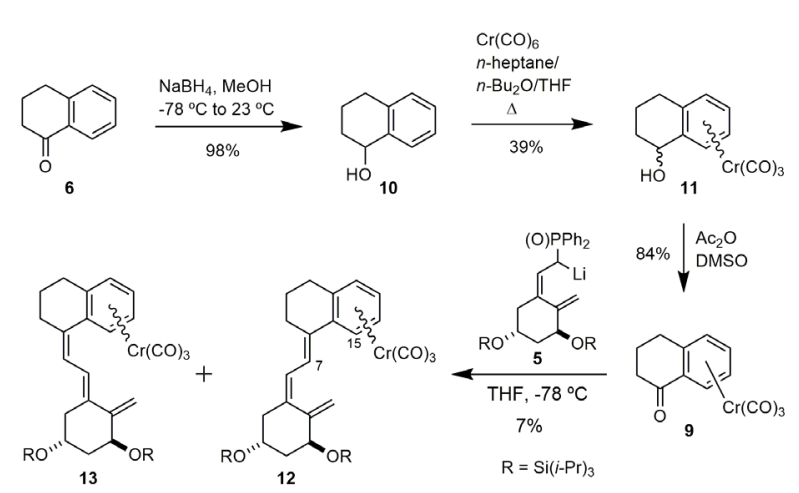

Figure 3 - Effect of $\mathrm{Cr}(\mathrm{CO})_{3}$-complexation on the WittigHorner coupling.

by vitamin D-previtamin D equilibration during thermal sigmatropic rearragement. Deprotection of $\mathbf{4 b}$ (TBAF, THF) gave the corresponding vitamin D compound (95\%), which equilibrates with its previtamin $\mathrm{D}$ form on standing in $\mathrm{CDCl}_{3}$ as determined by ${ }^{1} \mathrm{H}-\mathrm{NMR}$ (vitamin $\mathrm{D} /$ previtamin $\mathrm{D}$ ratio $=1 / 2.2$, equilibration time $=120 \mathrm{~h}$, room temperature).

\section{CONCLUSIONS}

In summary, we have demonstrated that the dienyne route is suitable for the synthesis of vitamin D analogs with aromatic D-ring for biological evaluation. The key feature of the synthesis is the complexation of the aromatic D-ring with $\mathrm{Cr}(\mathrm{CO})_{3}$ to partially release conjugation with the previtamin triene system, thus allowing the thermal sigmatropic $[1,7]-\mathrm{H}$ shift to generate the desired vitamin $\mathrm{D}$ form. The formation of $\mathrm{Cr}(\mathrm{CO})_{3}$ complexed vitamin $\mathrm{D}$ derivatives opens the way to a novel class of VDR-ligands for biological testing.

(Spectral data $\left({ }^{1} \mathrm{H}\right.$ and ${ }^{13} \mathrm{C}$ NMR) of all compounds - Supplementary Material)

\section{ACKNOWLEDGMENTS}

We thank Xunta de Galicia (project GPC2014/001) and for financial support. Silvina Eduardo thanks the Spanish MEC for a fellowship. Rita Sigüeiro

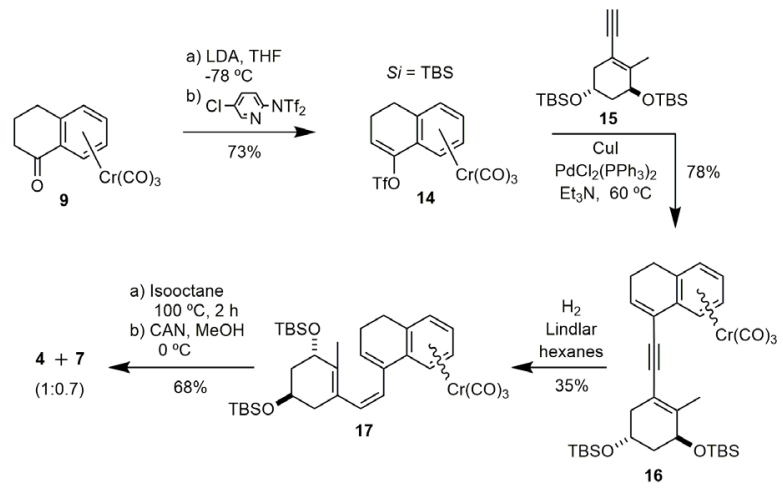

Figure 4 - Effect of $\mathrm{Cr}(\mathrm{CO})_{3}$-complexation on the sigmatropic $[1,7]-\mathrm{H}$ shift.

thanks Xunta de Galicia for a post-doctoral fellowship (Axudas posdoutorais, plan I2C, mod B).

\section{REFERENCES}

BOUILLON R, OKAMURA WH AND NORMAN AW. 1995. Structure function relationships in the vitamin D endocrine system. Endocr Rev 16: 200-257.

CASTEDO L, MOURIÑO A AND SARANDESES LA. 1986. Palladium-catalyzed synthesis of dienynes related to vitamin D from enoltriflates. Tetrahedron Lett 27: 15231526.

EDUARDO-CANOSA S, FRAGA R, SIGÜEIRO R, MARCO M, ROCHEL N, MORAS D AND MOURIÑO A. 2010. Design and synthesis of active vitamin D analogs. J Steroid Biochem Mol Biol 121: 7-12.

EVANS RM. 1988. The steroid and thyroid hormone receptor superfamily. Science 240: 889-895.

FELDMAN D, GLORIEUX FW AND PIKE JW. 1997. Vitamin D. Academic, New York, 1997.

FELDMAN D, PIKE JW AND ADAMS JS (Eds). 2011. Vitamin D, $3^{\text {rd }}$ ed., London, U.K. Academic Press.

GLEBOCKAAAND CHIELLINI G. 2012. A-Ring analogs of 1,25- dihydroxyvitamin $\mathrm{D}_{3}$. Arch Biochem Biophys 523: 48-57.

KENSLER TW, DOLAN PM, GANGE SJ, LEE JK, WANG Q AND POSNER GH. 2000. Conceptually new deltanoids (vitamin D analogs) inhibit multistage skin tumorigenesis. Carcinogenesis 21: 1341-1345.

KLIEWER SA, UMESONO K, MANGELSDORF DJ AND EVANS RM. 1992. Retinoid X receptor interacts with nuclear receptors in retinoid acid, thyroid hormones and vitamin $\mathrm{D}_{3}$ signaling. Nature 355: 446-449.

LINCOLN MR ET AL. 2010. A ChIP-seq defined genomewide map of vitamin D receptor binding: associations with disease and evolution. Genome Res 20: 1352-1360. 
LYTHGOE B. 1980. Synthetic approaches to vitamin D and its relatives. Chem Soc Rev 9: 449-475.

MA Y, YU WD, HIDALGO AA, LUO W, DELANSORNE R, JOHNSON CS AND TRUMP DL. 2013. Inecalcitol, an analog of $1,25 \mathrm{D}_{3}$, displays enhanced antitumor activity through the induction of apoptosis in a squamous cell carcinoma model system. Cell Cycle 12: 743-752.

MOLNAR F ET AL. 2011. 1 $\alpha, 25(\mathrm{OH})_{2}$-3-Epi-vitamin $\mathrm{D}_{3}$, a natural physiological metabolite of vitamin $\mathrm{D}_{3}$ : its synthesis, biological activity and crystal structure with its receptor. PLoS ONE 6(3): e18124.

NORMAN AW. 1979. Vitamin D the Calcium Homeostatic Steroid Hormone, Academic Press, New York.

OTERO R, SEOANE S, SIGÜEIRO R, BELORUSOVA AY, MAESTRO MA, PÉREZ-FERNÁNDEZ R, ROCHEL N AND MOURIÑO A. 2016. Carborane-based design of a potent vitamin D receptor agonist. Chem Sci 7: 1033-1037.

PLUM LA AND DELUCA HF. 2010. Vitamin D, disease and therapeutic opportunities. Nat Rev Drug Discovery 9: 941955.

PLUM LA, PRAHL JM, MA X, SICINSKI RR, GOWLUGARI S, CLAGETT-DAME M AND DELUCA HF. 2004. Biologically active noncalcemic analogs of 1 $\alpha, 25$-dihydroxyvitamin D with an abbreviated side chain containing no hydroxyl. Proc Natl Acad Sci USA 101: 6900-6904.
SCHMALZ HG, MILLIES B, BATS JW AND DÜRNER G. 1992. Diastereoselective complexation of temporary chyrally modified ligand: enantioselective preparaton and configurational assignment of synthetically valuable $\eta^{6}$-tricarbonylchromium-1-tetralone derivatives. Angew Chem Int Ed 31: 631-633.

SONOGASHIRA K. 1998. Metal-catalyzed cross-coupling reactions. Diederich F and Stang PJ (Eds), Wiley-VCH: Weinhemin, Germany, p. 203-229.

VERLINDEN L, VERSTUYF A, VAN CAMP M, MARCELIS S, SABBE K, ZHAO XY, DE CLERCQ P, VANDEWALLE M AND BOUILLON R. 2000. Two novel 14-epi-analogues of 1,25-dihydroxyvitamin $\mathrm{D}_{3}$ inhibit the growth of human breast cancer cells in vitro and in vivo. Cancer Res 60: 2673-2679.

YAMADA S AND MAKISHIMA M. 2014. Structure-activity relationship of nonsecosteroidal vitamin $\mathrm{D}$ receptor modulators. Trends in Pharmacol Sci 35: 324-337.

ZHAO Q, CURRAN DP, MALACRIA M, FENSTERBANK L, GODDARD JP AND LACÔTE E. 2012. NHCCatalyzed chemo- and regioselective hydrosilylation of carbonyl derivatives. Synlett 2012: 433-437.

\section{SUPPLEMENTARY MATERIAL}

Spectral data $\left({ }^{1} \mathrm{H}\right.$ and ${ }^{13} \mathrm{C}$ NMR $)$ of all compounds. 